\title{
CONTRIBUCIÓN Á LEXICOGRAFÍA HISTÓRICA DO GALEGO: O VOCABULARIO DE PAYZAL
}

\author{
MANUEL FERREIRO \\ Universidade da Coruña
}

Constitúe xa un lugar común o aserto sobre o permanente retraso dos estudios filoloxico-linguísticos a respecto do galego, a pesar do espectacular avance realizado nas últimas décadas. Ora ben, se aspectos como a dialectoloxía ou a formalización gramatical da nosa lingua contan hoxe con estudios cada vez máis serios e completos, a lexicografia histórica segue a reflectir as carencias causadas pola permanente anormalidade sociolingüística e cultural que o galego sufriu a partir do esplendoroso período trobadoresco.

Non podía acontecer doutro modo: mentres as 'grandes' linguas románicas (portugués, español, italiano, francés) comezan desde cedo o labor de fixación e estudio dos seus propios materiais lingüísticos, o galego sómese nun longo período de escuridade até desembocar nun século XIX en que, lenta e dificultosamente, se vai iniciando o camiño da súa recuperación.

Non obstante, o período dos Séculos Escuros non é un deserto absoluto neste ámbito: xa desde o século XVI contamos con escasos, mais valiosos materiais, cuxa publicación sen dúbida contribuíu grandemente ao coñecemento e profundización na traxectoria lingǘstica do romance de aquén-Miño a partir dos derradeiros anos da etapa medieval. Referímonos, é claro, ás 'humildes' anotacións léxicas do Bacharel Olea ${ }^{1}$ e aos importantes traballos de Cornide ${ }^{2}$, Sobreira ${ }^{3}$ e Sarmiento ${ }^{4}$, xa en pleno século da Ilustración ${ }^{5}$.

1 Vid. J. Filgueira Valverde, "El primer Vocabulario Gallego y su colector, el bachiller Olea (c. 1536)", Cuadernos de Estudios Gallegos, Santiago de Compostela, VII, 1947, pp. 591-608.

${ }^{2}$ Vid. C. Martínez-Barbeito, "Don José Comide y su 'Catálogo de palabras gallegas'", Boletín de la Real Academia Gallega, La Coruña, XXVII, 309-320, 1956, pp. 291349.

Cadernos de Lingua 9 (1994), 55-88 
No século XIX, co Rexurdimento prodúcese o salto desde os materiais dispersos e os vocabularios aos dicionarios, que se lucran, xa a partir da primeira contribución de Francisco Javier Rodríguez ${ }^{6}$, dos extraordinarios traballos do padre bieito, con maior ou menor fortuna ${ }^{7}$. Así, durante este mesmo século, aínda aparecerán os dicionarios de J. Cuveiro Piñol $^{8}$ e, sobre todo, o de M. Valladares Núñez ${ }^{9}$.

Agora ben, as condicións socioculturais en que foron realizadas estas obras $^{10}$, xunto coa deficiente formación lingüística dos seus autores, aconsellan un labor de revisión en que o profesor Pensado está empeñado desde hai anos:

"Una de las tareas más apremiantes de la lexicografia gallega, y previa a cualquier otra investigación seria sobre la misma, es la revisión crítica de todos los materiales que se han ido acumulando en sus diccionarios a lo largo de la historia" ${ }^{11}$.

${ }^{3}$ Vid. Fr. Juan Sobreira, Papeletas de un Diccionario Gallego. 1. Texto. Edición y estudio crítico por J. L. Pensado, Orense, Instituto de Estudios Orensanos "Padre Feijoo", 1979.

${ }^{4}$ Na maioría das obras 'linguisticas' de Frei Martín Sarmiento, todas elas paciente e progresivamente editadas na Universidade de Salamanca por J. L. Pensado, documéntanse valiosos materiais lexicográficos, especialmente en Colección de Voces y Frases Gallegas (1970), Catálogo de Voces y Frases de la Lengua Gallega (1973), e Catálogo de voces Vulgares y en especial de Voces Gallegas de diferentes Vegetables (1984).

Para unha perspectiva xeral sobre a lexicografia durante este século, vid. J. L. Pensado, "La lexicología gallega en el siglo XVIII", in D. Kremer / R. Lorenzo: Tradición, actualidade e futuro do galego. Actas do Coloquio de Tréveris, Santiago de Compostela, Xunta de Galicia, Conselleria de Cultura, 1982, pp. 85-93.

1863.

6 Diccionario Gallego-Castellano, La Coruna, Imp. del Hospicio Provincial,

7 J. L. Pensado comezou unha extraordinaria tarefa de revisión dos materiais lexicográficos galegos, que continuará en futuros traballos, xa anunciados. Produto desa investigación é o volume Contribución a la crítica de la Lexicografia Gallega. I. El Diccionario Gallego-Castellano de $F$. J. Rodriguez y su repercusión en la lexicografia gallega, Universidad de Salamanca, 1976.

8 Diccionario Gallego, Barcelona, Tip. de Ramirez y Cia, 1876.

9 Diccionario Gallego-Castellano, Santiago, Tip. del Seminario Conciliar Central, 1884.

10 Unha visión xeral da lexicografia decimonónica galega pode verse en J. L. Pensado, "La lexicografia gallega decimonónica", in Coloquio de Lexicografia, Anexo 29 de Verba, Universidade de Santiago de Compostela, 1988, pp. 49-55.

11 Cfr. Contribución a la critica..., op. cit., p. 3. Xa indicamos que J. L. Pensado pensa ampliar cronoloxicamente o seu labor critico (vid. n. 7), pero mesmo a revisión dos últimos dicionarios publicados é unha tarefa urxente e necesaria, como demostra o traballo de R. López Fernández ("Sobre os diccionarios galegos", A Trabe de Ouro, Santiago de Compostela, 3, Xullo/Agosto/Setembro, 1990, pp. 395-409). 
Mais os dicionarios non son as únicas fontes para o estudio da lexicografia galega decimonónica: consérvanse os manuscritos dalgúns vocabularios, na súa inmensa maioría de moi limitada extensión e concepción, que tamén constitúen importantes materiais para 0 estudio histórico do galego ${ }^{12}$.

O glosario de Payzal, cuxo manuscrito se conserva na Real Academia Galega, é un deses exemplos de traballo lexicográfico que debemos sumar ao realizado na súa maior parte por escritores aos que a Academia encargou a recollida de léxico popular para a redacción do que ía ser o grande Dicionario Galego-Castelán da institución ${ }^{13}$.

Este vocabulario consérvase encadernado nun volume $(26,6 \times 17,5$ $\mathrm{cm}$.) que tamén inclúe o elaborado por Leiras Pulpeiro ${ }^{14}$, co título $\mathrm{Vo}$ cabularios gallegos castellanos no lombo. As dúas primeiras páxinas, non pertencentes ao manuscrito orixinal, presentan, sucesivamente, os titulos Vocabularios Gallegos e Vocabulario / Gallego-Castellano / por / $B$. Vicente Payzal. O ms. propiamente dito $(21,2 \times 15 \mathrm{~cm}$.) comeza co título Vocabulario / Gallego-Castellano / de Payzal (1r.). No verso desta páxina (1v.) aparecen os únicos datos (con tinta xa moi borrosa e letra de moi dificil lectura) que actualmente coñecemos acerca da autoría e personalidade do autor do glosario: ¿Fueron? echos y ¿estaba? de letra / del Licenciado D. Benito Vicente Payzal / Son hoy de Jose Baltar (segue rubrica).

A continuación, antes de $2 \mathrm{r}$. aparecen encadernadas no volume seis folliñas ( $16 \times 20,2 \mathrm{~cm}$.) coas voces Atadeira, Atadura e Asimbre na primeira (coa observación copiado escrita a lapis) e máis de novo Atadeira na quinta, quedando en branco as intermedias e a sexta.

12 Limitándonos ao século XIX, podemos citar os anónimos Dialecto de Galicia, ms. da Real Academia de la Historia de Madrid (vid. I. L. Pensado, "Un vocabulario gallego del siglo XIX", in VV.AA. Homaxe a Ramón Otero Pedrayo no LXX aniversario do seu nacimento, Vigo, Galaxia, 1958, pp. 275-286), Vozes Gallegas, ms. na Biblioteca Nacional de Madrid, editado por J. Leite de Vasconcelos (vid. "Estudos de Filologia Gallega", Revista Lusitana, Lisboa, VII, 1902, pp 198-210), ou a anónima Traducción de algunas voces, frases $i$ locuciones gallegas, especialmente de Agricultura, al castellano, de próxima publicación (vid. J. L. Pensado, "Sobre un nuevo diccionario gallego", in El gallego, Galicia y los gallegos a través de los tiempos (Ensayos), La Coruña, La Voz de Galicia, 1985, pp. 65-67.

${ }^{13}$ No incompleto Diccionario Gallego-Castellano (ata a palabra cativo), publicado na Coruna entre 1913 e 1928, aprovéitanse os materiais que diversos autores e escritores galegos foron recollendo polo territorio galego. Para esta cuestión, vid. X. M. Jiómez Clemente / X. B. Arias Freixedo, "O 'Vocabulario gallego-castellano' de Eduardo Pondal", in Cadernos de Lingua, Real Academia Galega, 2, $2^{\circ}$ trimestre, 1990, pp. 18-20.

14 Vid. I. Pérez Pascual / C. Tato Garća, "O vocabulario de Leiras Pulpeiro", Verba, 13, 1986, pp. 237-267. 
Tampouco pertencen ao ms. orixinal dúas pequenas follas $(10 \times 8$ $\mathrm{cm}$.), grampada no bordo superior de $2 \mathrm{r}$. a primeira, co texto Letra $D . /$ Payzal No se copio mas / que hasta esta letra / inclusive, e a segunda, escrita en vertical, encadernada entre 3v. (letra A) e 4r. (letra C), coa observación El Diccionario no trae / la letra $\underline{B}$.

Así pois, o manuscrito está constituído por 29 follas sen numerar, escritas por ambas as dúas caras, repetindo a folla $28 \mathrm{r}$. os vocábulos da letra $Y$, coa mesma letra dos dous folios iniciais antes descritos.

Non conseguimos achar datos acerca da personalidade de José Baltar, o posuidor do manuscrito que entregaría á Academia para o seu aproveitamento na redacción do dicionario ${ }^{15}$. Por outra parte, a persoa do Licenciado Benito Vicente Payzal tamén nos é totalmente descoñecida, mais a letra, decimonónica con trazos dezaoitescos, suxírenos que, a diferencia dos escritores convocados pola institución académica para a realización de papeletas lexicográficas, agora estamos perante un texto dalgún curioso ou erudito situado cronoloxicamente entre os séculos XVIII e XIX. Xeograficamente, o léxico (e tal vez o autor) responde, polas súas características linguístico-dialectais, ao bloque occidental, como mostran os frecuentes casos de seseo (implosivo e explosivo) presente en numerosos vocábulos ${ }^{16}$, mesmo no español, así como a solución -án, e o seu plural -ns, para a terminación latina -anu/-ana.

No vocabulario contabilizanse arredor de 900 entradas, agrupadas por letras sen orde alfabética interna (faltan as correspondentes a $B, I^{17}, T$ e $Z$ ), que aumentan se considerarmos a presencia de sinónimos ou variantes na mesma entrada.

Practicamente non existe ningunha definición strictu sensu; trátase simplemente de explicacións que matizan o vocábulo xunto coa tradución correspondente ao español, ás veces de marcado carácter arcaico ou dialectal en relación á sincronia actual. Por outra parte, as entradas non van acompañadas de observacións gramaticais, agás no caso de Mortal. $\mathrm{Na}$ realidade, fóra do valor histórico e da aportación dalgúns vocábulos non dicionarizados aínda hoxe ${ }^{18}, \mathrm{o}$ valor do glosario payzaliano é relativo xa

15 Como efectivamente aconteceu, pois o recurso a Payzal para aboar algunhas roces ou acepcións aparece de cando en vez. Cfr., por exemplo, a segunda acepción do vocábulo alcoba no Dicionario académico, onde se copia literalmente a definición de Payzal, puntualmente indicada, por outra parte.

16 Tamén aparece algún caso de contraseseo, como maceira por maseira.

17 Incluidas nas letras $H \mathrm{e} / \mathrm{ou} Y$.

18

Entre outros, non achamos recollidos vocábulos como Monciño (do diminutivo lat. de Monachu), Leba e trag[u]e (cfr. Leva-e-traz, "Pessoa intrigante, mexeriqueira", voz trasmontana citada por Cândido de Figueiredo, Grande Dicionário da Lingua Portuguesa, Lisboa, Livr. Bertrand, 1978, 15 ed.), Queimosa (cfr. Queimada, "Pústula carbunculosa", voz de Chaves, ibid.), Gurumelo (mais si Agurumelar) ou Mexoca; ademais, ao longo do 
que, alén da súa pobreza técnica, presenta bastantes erros, detectábeis na súa maioria, ademais de castelanismos evidentes e vocábulos de dubidoso carácter galego ${ }^{19}$.

De todos os xeitos, o Diccionario da Real Academia Galega aproveita e recolle, loxicamente, os materiais fornecidos por Payzal, podéndose comprobar a súa influencia en bastantes definicións ou acepcións (vid. Abarasa (sic), Alforja, Alcoba, Alma, Almoda, Almendrillas, Amormeirarse, Aro, Cadeira, Cagarria, Caixón, Caldeado ou Carrexón), así como a referencia directa á autoridade de Payzal para aboar algunhas voces (Alcoba, Aljóujere ou Cagarría).

A partir do dicionario académico, sen entrarmos a considerar algunhas aportacións lexicográficas menores, os materiais de Payzal parecen ter sido revisados por Leandro Carré Alvarellos (véxanse, por exemplo, voces como Colludo, Leitaruga ou Monlla no seu dicionario ${ }^{20}$ ) e mais por Eladio Rodriguez González, que os incorporou dun modo bastante sistemático ata chegar á letra $\mathrm{F}$, a partir da cal xa non aparece ningunha referencia ao vocabulario payzaliano. É por isto que a influencia das definicións de Payzal está presente en bastantes voces do dicionario enciclopédico $^{21}$ (Cotexar, Cuña, Currucho, Escalo, Fol, etc.), ao tempo que se cita directamente a súa procedencia noutras (Caparuza, Desangrar, Desumar, Emponla, Encabezar, Enlagar, Encertarse, Entrecocedura, Esgalletar, etc.); mais a partir da letra $\mathrm{F}$ apréciase a ausencia de bastantes vocábulos que, a pesar de todo, reaparecen en dicionarios modernos que extraen o seu léxico de fontes populares ou dialectais.

De todos os xeitos, despois do monumental traballo de Eladio Rodríguez, evidentemente consultado e aproveitado por todos os dicionaristas posteriores, o manuscrito de Payzal ficou no esquecemento, provocando que bastantes vocábulos ficasen sen dicionarizar e algunhas acepcións sen recoller, mesmo en traballos lexicográficos de intención globalizadora $^{22}$.

vocabulario encóntranse variantes ou acepcións non dicionarizadas, como Amañencer (produto do cruzamento entre Amañecer e Amencer), Escoupre (do lat. Scalpru), Esquensa (de Esquenser), Gazo (o mesmo que Gacio), Rancearse e Ranceheira (a carón de Randearse e Randeheira), etc.

19 Véxanse, por exemplo, Afibillar, Manoxo, Señal ou Sambijuelas, ademais doutros como Chaquete e Xaquete, Copete, Estanquillo ou Falla.

${ }^{20}$ Manexamos a $4^{\mathrm{a}}$ ed., de 1972 (Diccionario Galego-Castelán e Vocabulario Castelán-Galego, Coruña, Ed. Moret).

${ }^{21}$ Cfr. Diccionario Enciclopédico Gallego-Castellano, Vigo, Ed. Galaxia, 19581961 ( 3 vols.).

22 Como se pode comprobar no confronto, por exemplo, co de Isaac Alonso Estravis, Dicionário da Língua Galega, Madrid, Alhena Ediciones, 1986 (3 vols.). 
Para a presente edición utilizamos o sistema habitual neste tipo de textos, organizando alfabeticamente as entradas (suprimindo aquelas repetidas sen ningunha alteración), a partir da anárquica disposición orixinal dentro de cada letra, intentando sempre manter o máximo respecto á lingua do autor, mesmo nos seus aspectos gráficos, ao tempo que anotamos escrupulosamente, por medio dos signos habituais ou con notas a pé de páxina, todo tipo de alteración que consideramos pertinente realizar por se tratar de lapsos ou erros ${ }^{23}$, asi como calquera outra información que consideramos pertinente sobre certos vocábulos, independentemente do seu carácter galego ou español ${ }^{24}$. Ao mesmo tempo, engadimos (sempre utilizando os corchetes) as referencias internas que nos pareceron oportunas para unha máis fácil e completa consulta do vocabulario.

Canto ás definicións ou explicacións en español, separadas no manuscrito da voz definida por medio dun punto, incluímolas entre parénteses, modernizando a puntuación e desenvolvendo por medio da cursiva as frecuentes abreviaturas que, etc. ou para, ao mesmo tempo que regularizamos a acentuación, inexistente no caso das voces galegas e arbitraria nas voces en español ${ }^{25}$.

\section{[-A-]}

ABARASA (que se pone a la madeja para que no se enrrede, como es costal): Cuenda.

ABOLTO: Rebuelto. Heciento.

ABRAZADEIRAS: Abrazaderas.

ABRAZADEIRAS (de baúl, arca, etc.): Barretas.

ABRÓTEGAS (parece ser el gamón, o macho o hembra): Gamón.

ACARIÑAR: Acariciar.

ACARIÑAR (con fiestas o manoseando): Popar.

ACARÓN (por imediato): A carona.

ACEXAR: Acechar. Avizorar.

23 Intentamos respectar as características lingulisticas do autor (seseo ou contraseseo, por exemplo), mais enmendamos e/ou indicamos aqueles casos que parecen ser unha deficiente interpretación (copia tal vez dun manuscrito anterior) de fácil explicación paleografica (Atriceses por Arriceses, Tupetrape por Tripetrape, etc.), lapsos interpretativos (Tacón por Tocón, Azotacallos por Azotacalles, etc.) ou debidos ao galego (Cuberta ou Padrasto). Por outra parte, respectamos as entradas con formas femininas, diminutivas ou plurais.

24 Debido ao carácter arcaico ou dialectal de bastantes voces do espanol, introducimos aclaracións cando estas non aparecen nas edicións modernas do dicionario da Real Academia Española.

${ }^{25}$ Alén disto, introducimos a letra cursiva para indicar algunha voz galega utilizada nas explicacións en español. 
ACUGULADO/ACUGULO: Colmado.

ACUGULAR: Colmar.

[ACUGULO: Vid. ACUGULADO].

ADIVIÑAR: Adivinar.

ADIVIÑO (que se haze por los huesos ${ }^{26}$ de los animales): Espatuloman$\mathrm{cia}^{27}$.

ADRO: Atrio.

ADRO (el muro o pared que lo divide o cierra): Ba[r]bacana.

AFIBILLAR: Hebillar.

AFOZIÑAR/CAER DE FOCIÑOS: Hocicar.

AGÜEIRO (hecho en el muro): Clavigera.

[AGULLA: Vid. CORTAMAR].

AGULLA (de puente): Espolón.

AGULLA (de puente): Tajamar.

AGURUMELAR (las cebollas, ajos, centeno etc.): Grillar. Tallecer. Entallecer.

ALA: Ala. Alón.

ALACENA (para abejas): Armario.

ALAZENA (en que se guardan pucheros, cazuelas y otras cosas de barro): Barrera.

ALBÉRCHEGO: Albaricoque.

ALCOBA (sobre la escalera y otra parte semexante): Camareta.

ALENTO: Aliento.

ALENTOS (por fuerzas): Vigor.

ALFORJA (que trahen colgada los pobres para recoger la limosna): Burjaca.

ALGODÓN (que hay debajo de las camas, arcas, etc.): Tamo.

ALGODÓN (que sale al tejer): Tamo.

ALLO: Ajo.

ALMA (los palos o cañas que cruzan la colmena): Trencas.

ALMENDRILLAS: Pendientes. Perendengues.

ALMODA: Almoada.

ALMODA (para palillar): Mundillo.

ALMORSO: Almuerzo.

ALODADO (o rebuelto el vino, agua, etc.): Heciento.

[ALRROTAR: Vid. ARROTAR].

ALRROTO/ARROTO: Eructo. Regüeldo.

ALUGAR: Alquilar. Arrendar. Alogar. Alugar.

ALUGUEIRO: Arrendamiento.

${ }^{26}$ No ms. o $h$ corrixe un $g$ anterior.

27 Ms. Espatulamancia. 
ALXÓUXERE: Cascabel.

ALXÓUXERE (lo que tiene dentro para que suene): Escrupulillo.

AMACHAMARTELO: A machamartillo.

AMAINAR (el viento): Amainar. Calmar.

AMAINAR (la cólera): Amainar. Aflojar. Sosegar.

AMANSAR: Amansar. Domesticar.

AMAÑADO: Acostumbrado. Amañado.

AMAÑARSE: Amañarse.

AMAÑENCENDO: Amaneciendo.

AMAÑENCER: Amanecer.

AMAÑOS: Amaños. Aparejos.

AMARRALLA: Cordel.

AMARRALLINAA: Cordelillo. Cordelico. Cordelito.

AMARRAR (por detener o asegurar): Asir.

AMARRAR (quando se habla de embarcaciones, o asegurar con cuerdas): Amarrar. Atar con cuerdas.

AMASAR: Sobar.

AMASAR (el pan): Heñir.

AMEDRENTADOR (que pone grimo o miedo): Terrifico.

AMEIXA (marisco): Almeja.

AMENTA (yerba): Mienta. Yerbabuena.

AMOADO (para $\mathrm{h}[\mathrm{a}] z e r$ ostias o viscochos): Batido.

AMOLAR: Amolar. Afilar.

AMORMEIRADO (cargado de la cabeza): Azorrado.

AMORMEIRARSE (cargarse de cabeza): Azorrarse.

ANGARILLAS: Angarillas. Árguenas.

ANGASO: Rastrillo. Mielga.

ANIÑADOR (el huebo que se deja para ello): Ponedero.

APUPAR: Popar.

APURRAR: Azuzar.

AREA: Arena.

AREA (pedacito pequeño ${ }^{28}$ de piedra, teja, etc.): China.

AREA (que entra en el zapato y lastima el pie): Escrúpulo.

ARGOLA: Argolla.

ARGOLA (para candado, etc.): Armella.

ARMADILLA: Armadijo.

ARO (de cesto, caldero, etc.): Aro.

ARO (del pozo o la guarnición ${ }^{29}$ de su boca): Brocal. Puteal ${ }^{30}$.

${ }^{28}$ Ms. pequeno.

29 Palabra de lectura dubidosa, pero a definición do histórico Dicionario da Real Academia Galega parece confirmar esta lección ("Brocal. La guarnición de la boca de un pozo", cfr. s.v. ARO). 
ARQUEAR (de cansado, y comunmente el caballo): Yjadear.

ARREMETEDURA/ARREMETEMENTO: Amago.

[ARRETEMENTO: Vid. ARREMETEDURA].

ARREMETER (una persona a otra): Amagar.

ARRENDO: Arrendamiento. Arriendo.

ARRÓ: Acirate.

ARROLAR: Mecer.

ARROTAR/ALRROTAR: Eructar.

[ARROTO: vid. ALRROTO].

ARRUPIARSE (los cabellos, por espanto, terror, miedo, etc.: Erizarse los cabellos. Espeluznarse.

ARTES (especie de red): Xábega o Xábeca.

ÁS DÚAS MANS (dar un golpe): A manteniente.

ÁS MA[N]S ${ }^{31}$ CHEAS (lo que se coje o da con amba[s] juntas: Almorzada.

ASA: Asidero. Asidilla.

ASCUA: Ascua. Brasa.

ASEXAR: Acechar. Amaitinar.

ASIMBRE (para fornar sobre él la bóveda): Cimbra.

ASIRRAR: Azuzar.

ASPA (en lo escrito): Tachón.

ASPAR (lo escrito): Tachar.

ASUBIAR: Silbar.

ASUBÍO: Silbato.

ATADEIRA (de paja con que se aprieta el manojo): Tramojo.

ATADURA (de paja para el manojo): Tramojo.

ATALLO (camino que va por el atajo): Trocha.

ATURUXAR: Baladrar.

ATURUXO: Baladro.

\section{$[-\mathrm{C}-]$}

CABAR (hazer riegos o dar cabaduras para señalar por propio algún terreno o para otro fin): Amelgar.

30 "Brocal del pozo fatidico con una Ara encima, donde se ponian supersticiosamente los Jueces, a fin de que la Diosa Themis les inspirasse las sentencias [...]" (cfr. Diccionario de la Lengua Castellana, en que se explica el verdadero sentido de las voces, su naturelaza, y calidad, con las phrases, o modos de hablar, los proverbios, o refranes, $y$ otras cosas convenientes al uso de la lengua [... Compuesto por la Real Academia Española, En Madrid. En la Imprenta de la Real Academia Española: Por los Herederos de Francisco de el Hierro, vols. I-VI, 1726-1739). En adiante, citarémolo como Dic. Aut.

31 A forma mas, ainda que posibel, parece un erro de escrita por mans, en coherencia co modelo linguístico dialectal do autor (véxanse máis adiante gran e grans). 
CABAR (sacar a las viñas la tierra que se arrimó a las cepas o escarnar éstas algo alrrededor): Alumbrar.

CABASA: Calabaza.

CABASO (calabaza grande para vino o agua): Calabaza. Calabazo.

CABASO (para comer).

CABEZADA (golpe dado con la cabeza, especialmente una contra otra): Morrada.

CABRITO (de más de seis mes[es], que no llega al año: Chibato. Choto.

CABRITO (u otro animal de un año): Añal.

CACHAS (de la rueca): Costillas.

CACHAS (de la rueca en que se pone lo que se hila): Rocadero.

CACHEAR: Registrar.

CACHOS (de teja o qualquier especie de losa): Tejoleta. Tejuela.

CACHOURIZO; Erizo.

CADEIRA (el hueso ${ }^{32}$ de ella en el animal): Babada.

CADILLO (porción de hilos de la urdidumbre): Cadillo. Portada.

CADRADO (cosa quadrada): Anguloso.

CADRIL (el güleso de él en el animal): Babada.

CAER DE FOCIÑOS: Hocicar.

CAGALLA: Cagar[r]uta.

CAGARRÍA (en las caballerías): Viarasa.

CAIREL: Galón. Bastoncillo.

CAIXÓN: Cajón.

CAIXÓN (dentro de que se ponen la lavanderas): Banca.

CALDEADO (encendido que heche chispas o parezca blanco): Enalbado.

CALDIL: Candil.

CALDO, SACAR O CALDO: Escudillar.

CAMALLÓN: Caballón.

CAMALLÓN: Camellón. Camella.

CAMARIÑA (alcoba sobre la escalera, etc.): Camareta.

CAMIÑO: Camino.

CAMIÑO (donde se juntan tres): Trivio.

CAMIÑO (el que es atajo o va por el atajo): Trocha.

CAMIÑO (que tiene altos y bajos, o es desigual): Batidero.

CAMIÑO ESTREITO: Trocha.

CAMIÑOS (tres caminos donde se juntan): Trivio.

CAN (animal): Perro.

CAN (perrillo pequeño): Gozque.

CANASTRA (con su tapadera): Excusabaraja.

CANDIL (el que se pone dentro del otro): Candileja.

${ }^{32}$ No ms. o $h$ corrixe un $g$ anterior. 
CANGA: Horca.

CANGA: Tarangallo.

CANGAR (las casas para texarlas): Xabalonar. Xabalconar.

CANGO: Xabalón. Xabalcón.

CANGOS: Asnas $^{33}$.

CANGREXO: Cangrejo.

CANGREXO (de río): Astaco.

CANIZO: Zarzo.

CANO (de berza): Bohordo.

CANSIÑO: Perrillo.

CANTAR DOS GALOS ${ }^{34}$ (por ser la media noche): Gallicinio ${ }^{35}$.

CANTEIROS (maderos sobre que están las vasijas): Poíno. Codales.

CAÑEIRA: Estercolero.

CAÑOTO (de árbol que queda después de cortado): Tocón ${ }^{36}$.

CAÑOTO (de berza): Bohordo.

CAPA (de molino): Muela. Corredera.

CAPAR: Capar. Labrar.

CAPARUZA/CAPILLO (de paja o de otra cosa): Gallaruza.

CAPEALSO: Dintel.

CAPILLADA (la cub[i]erta del zapato): Pella ${ }^{37}$. Pala.

CAPILLO (de capa o otra ropa): Capirote.

CARAFUNCHO: Divieso.

CARAMUXO: Nácara.

CARAMUXO: Caracol marino. Nácara.

CARANTOÑA: Carátula.

CARANTOÑA (fea): Carantamaula.

CARBALLO: Roble.

CARNEIRO: Carnero.

CARNEIRO (padre): Morueco.

CARPINTEIRO: Carpintero.

CARQUEIXA: Carrasca.

CARRANDEAR: Anadear.

CARREIRA: Carrera.

CARREIRO (el que se haze en la cabeza, quando se divide el cabello): Carrera.

33 Ms. Asnos.

34 Ms. gallos, seguramente por lapso producido a causa do cruzamento co español. Cfr. n. 41 .

${ }^{35}$ Ms. Gillicinio. Cfr. Gallicinio, no Dic. Aut., fronte ás edicións modernas do dicionario da Academia Española, onde só aparece Galicinio.

36 Ms. Tacón.

${ }^{37}$ Non conseguimos achar este vocábulo coa acepción esperada 
CARREXAR: Acarrear. Conducir.

CARREXÓN (que lleba el pan al horno y lo trahe): Anacalo.

CARRIZO (pájaro): Paro.

CASACA (de vestir): Sobrevesta. E[s]crocón.

CASAS (las de los palomares para anidar): Hornilla.

CASCA (de fruta u otra cosa): Cáscara.

CASCA (la interior de algunas frutas y legumbres, como de la almendra, guisante, etc.): Camisa.

CASCA (la que se sacó a la fruta): Monda.

CASPA (que se haze en las heridas): Escoriación.

CATAR (en la cabeza u otra parte): Espulgar.

CEBOLA: Cebolla.

CEBOLA (los hijos que hechan las viejas): Cebolleta. Babosa.

CEPO (de natividad): Nochebueno.

CESTA: Cesto.

CESTIÑO (de calzeta, costura, etc.): Tabaquillo. Tabaque.

CESTO: Cesta.

CESTO (de paja): Escriño.

CHABE: Llabe.

CHAQUETE $^{38}$ (juego): Tabla.

CHASCA (pájaro): Curruca.

CHEIRAR: Oler.

CHEIRAR (mal): Heder.

CHEIRAR (mal la carne): Husmar.

CHEIRO: Olfato.

CHEIRO (malo): Hedor. Hediondez. Hedentina.

CHEIRO (malo que tienen las carnes añejas): Husmo.

CHÍCHAROS: Guisantes.

CHÍCHAROS (quadrados o medio quadrados): Dientes de muerto. Almortas. Guijas.

CHIFRE (de capador): Castrapuercas.

CHISMEIRO/CHISMOSO (persona que lleba y trahe cuentos): Otacusta.

[CHISMOSO: Vid. CHISMEIRO].

CHISTOSO (el que dice chistes con facilidad y gracia): Decidor.

CHOCA (gallina o otra ave): Llueca. Clueca.

CHOCA (prisión): Brete.

CHOCO (el güebo): Clueco.

CHOÍR: Cerrar. Tapar. Acotar.

CHOUSA: Cerradura.

38 Este vocábulo, presente nos dicionarios españois, é de dubidoso carácter galego, o mesmo que a variante Xaquete. 
CHOZA: Barraca.

CHUMBEIRA (rez para pescar): Esparavel.

CIFRA (en escribir, o escrivir con señales ${ }^{39}$ ocultos): Paleografia ${ }^{40}$.

COBA (tobo o sitio en que se recoje o cría el oso): Osera.

COBERTOR (peludo): Frazada.

COCA/COCO (que dan los robles): Bugalla. Agalla.

COCIÑA: Cocina.

[COCO: Vid. COCA].

COCÓN: Espectro. Fantasma.

COIRO: Piel.

COITELO $^{41}$ : Cuchillo.

COITELO (de punta corba): Falce.

COLGADO (lo que está en las tiendas para detener la luz o disminuirla, a modo de toldo o cenefa): Amaestradura.

COLLUDO (animal entero): Entero.

COLONDRO: parece ser Cohombro.

COMENZAR ${ }^{42}$ A FERBER: Alzar el hervor.

COMPANGO: Vianda.

COMPANGO (de miel, queso, azeite, etc.): Cundido.

CONFUSIÓN (de muchas especies): Tripitrape ${ }^{43}$.

CONGOSTRA: Trocha.

CONSELLO (por sedución o engaño): Prestigio.

COPETE $^{44}$ (pedazo de cuero del zapato que cae sobre el empeine del pie): Polaca ${ }^{45}$.

COQUETA (gorro de paño o cosa semexante): Becoquín. Galocha.

COQUETA (que se pone en la cabeza a las niños): Gambox.

CORBO (ave): Cuervo.

CORBO MARIÑO: Cuervo marino. Mergo.

CORDEL: Cordel.

CORDEL (de nivel o para nivelar): Tendel.

39 Ms. senales.

40 Ms. Paligrafia (?!).

41 Ms. coitello. Cfr. n. 34.

42 Mis. comerzar, por lapso de escrita.

${ }^{43}$ Ms. Tupetrape. A forma Tripitrape, que non aparece nas modernas edicións do dicionario da Real Academia Espantola, onde só consta unha forma equivalente Triquitraque, documéntase no Dic. Aut. como "Conjunto de trastos ridiculos, y de poca monta: y metaphoricamente se toma por la confusion de especies. Es del estilo familiar, y baxo" (cfr. s.v.).

44 Vid. n. 38.

45 "El copete ò vuelta del zapato, que cae sobre el empéine del pié, y suele ser de diferente colór" (cfr. Dic. Aut., s.v. POLACA). 
CORDELEIRO: Cordelero.

CORRE: Vardazca.

CORREAZO: Vardascazo.

CORRE-CANS: Perrero.

CORREDOIRA: Trocha.

CORREEIRO: Correero.

CORTAMAR/AGULLA: Espolón.

CORTELLO: Chiquero.

CORTIZA: Corcho.

CORTIZEIRA (árbol que da el corcho): Alcornoque.

COSEDURA (el pan que se cuece de una vez): Cochura.

COSEDURA (lo que se cuece, y el hecho de cocer): Cochura.

COSINEEIRA: Cocinera.

COSIÑEIRO: Cocinero.

COSTAL (de la madeja): Cuenda.

COSTRA (que se haze en las heridas): Escoriación.

COSTUREIRA: Costurera. Labrandera.

COTA (de cuchillo, nabaja, etc., que es la parte opuesta o contraria al filo): $\mathrm{Cazo}^{46}$. Recazo.

COTEXAR (por dar cornadas): Amurcar.

CROQUE (golpe dado con la cabeza): Morrada.

CUADA (caída): Tamborilada.

CUBERTA: Cubierta ${ }^{47}$.

CUBERTA (de mesa): Carpeta.

CUBERTA (que se pone sobre la silla y arreos de caballo de respeto, o después de apearse): Telliz.

CUBERTURA: Cub[i]erta.

CUME (de tejado): Caballete.

CUNCA: Hortera. Cuenca. Conca.

CUNCHA (la caballería que tiene una estrella en la frente): Careto. Careta.

CUÑA (pieza que hechan los sastres en la faldilla de la espalda del bestido para formar un pliegue): Falbalá.

CURANDEIRO: Medicastro.

CURRUCHO (sitio oculto): Ostugo.

CURTIDOR: Curtidor. Noquero ${ }^{48}$.

CURUXA: Lechuza.

\footnotetext{
46 Vid. n. 37.

47 Entrada repetida.

48 Entrada repetida.
} 
DEBAIXO DO BRAZO: Sobaco.

DEBANADOR: Devanador.

DEBANADORA: Devanadora.

DEBANAR: Devanar ${ }^{49}$.

DEBANDOIRA: Devanadera.

DECLARACIÓN (de guerra, el manifiesto para ello): Difidencia.

DENTADA: Tarascada.

DENTELLA: Faba. Haba.

DEREITO: Derecho.

DEREITO (por cara de tela, paño, etc.): Haz.

[DESANGRAR: Vid. DESUMAR]

DESEXADO: Deseado.

DESOCUPADO (ocioso): Valdío.

DESTERRO: Destierro. Confinación.

DESTERRO (político o de temor al desterrado): Ostracismo.

DESUMAR/DESANGRAR (la tierra, dar salida a las aguas): Avenar.

\section{$[-\mathrm{E}-]$}

EMBROLLA (de muchas especies diferentes): Tripitrape ${ }^{50}$.

EMPEZAR A FERBER: Alzar el herbor.

[EMPOLARSE: Vid. ESCALDARSE]

EMPONLA: Ampolla.

EMPONLA (de quemadura o de andar): Ampolla.

ENCABEZAR (los árboles): Acotar.

ENCONTRÓN (golpe que se da con el cuerpo): Empellón.

ENCRUCILLADA: Encrucijada.

ENCRUCILLADA (de tres caminos): Trivio.

ENCRUCILLADA (junta de tres caminos): Trivio.

ENFIADA (de hilo, seda, etc.): Hebra.

ENLAGAR (el lino): Embalsar.

ENLORDADO: Cazcarriento.

ENSERTAR (por empezar a gastar alguna cosa): Decentar.

ENSERTARSE (por llagarse el enfermo o el viejo, o entrecoserse el niño): Decentarse.

ENSILBEIRADURA: Barda.

ENSILBEIRAR: Bardar. 
ENSIRRAR: Azuzar.

ENTOBARSE (el conejo o otro animal): Amadrigarse.

ENTRECOSEDURA: Colisión.

ENTRECOSEDURA: Sahorno.

ENXURRO (de agua): Aluvión.

ERGUER: Levantar.

ESCALDARSE/EMPOLARSE: Ampollarse.

ESCALO: pare[ce] ser carpa.

ESCARRANCHARSE: Esparrancarse.

ESCOFADURA: Reconcomio.

ESCOFARSE: Reconcomerse.

ESCOITAR: Escuchar.

ESCOITAR/ACEXAR (con cuidado): Avizorar.

ESCORA: Tentemozo.

ESCORREDIZO (como la anguila, etc.): Escurridizo.

ESCORREDURA (lo que escurre de alguna vasija): Escurriduras.

ESCORREGADOIRO: Resbaladero.

ESCORREGAR: Escurrir. Resbalar.

ESCORRER (alguna vasija): Escurrir.

ESCOUPRE: Escoplo.

ESCUMA: Espuma.

ESCUMA (del jabón para hazer bolas): Bálago.

ESCUMA (gorda o abultada): Babaza.

ESCUMAR: Espumar.

ESFRANGULLAR: Migar.

ESGADELLADO (tener descompuestos los cabellos): Espeluznado.

ESGADUÑAR: Arañar. Arpar.

ESGALLETAR (hazer tiras o pedazos alguna ropa o otra cosa): Rasgar.

Arpar.

ESGARRO: Gargajo.

ESMOCAR: lo mismo que ESPABILAR.

ESPABILADEIRAS: Tijeras. Tenacillas. Tenacicas. Espabiladeras.

Despabiladeras.

ESPABILAR: Espabilar. Despabilar.

ESPADELA: Espadilla.

ESPADELAR (el lino): Espadar.

ESPANTALLO: Espantajo.

ESPANTALLO (de figura de hombre): Bausán.

ESPIGA (de mijo): Mazorca. Panoja.

ESQUENSA: Olvido. Descuido.

ESQUENSA (por tener un hijo): Desliz. Descuido.

ESQUENSER: Olvidar. 
ESQUINADO (lo que tiene quadras): Anguloso.

ESTADULLO: Estadoño. Estaca. Estadojo.

ESTALAR/ESTOIRAR/ESTOUPAR: Estallar.

ESTALO: Estallido.

ESTANQUE (de tabaco): Tabaquería.

ESTANQUEIRO (de tabaco): Tabaquero.

ESTANQUILLEIRO (de tabaco): Tabaquero.

ESTANQUILLO $^{51}$ (de tabaco): Tabaquería.

ESTEIRA: Estera ${ }^{52}$.

ESTEIRA (de cañas): Zarzo.

ESTEIRA (la tira o lista de que se haze): Pleyta.

ESTENDER: Tender.

ESTERCO (de palomas): Palomina.

[ESTOIRAR: Vid. ESTALAR].

ESTORNELA: Estornija. Tala.

ESTORNIÑO (ave): Estornino.

[ESTOUPAR: Vid. ESTALAR].

ESTRELLADA (la caballeria que tiene una estrella en la frente): Careto.

ESTREMA (división de guertas, hortalizas, etc.): Tablar.

ESTREMA: Ve ARRÓ.

ESTRIBEIRA (de zapatero): Tirapié.

ESTRIBO (de pared): Espolón.

ESTROBO: Escálamo.

ESTROBOS: Estrobos.

FABA: Faba. Haba.

FACER (burla, dando golpes o de otra suerte): Popar.

FACHEIRO (de pajas): Mellón.

FACHOS (la hoguera que hazen de señal): Almenara.

FACHUCO (de pajas): Mellón.

FACOCO o FACOCOS (por los me[n]drugos de pan o zoquetes que se dan de limosna): Gallofa.

FALLA $^{53}$ (el capillo que se pone a los ninos en la cabeza): Gambox.

FALTA (que hai en los sembrados): Estar amelgados.

FALTRIQUEIRA: Faldriquera. Faltriquera. Bolsillo.

51 Este vocábulo aparece no Dic. Aut. como diminutivo de Estanco. Vid. n. 38.

52 Esta entrada, incompleta e riscada, aparece tamén incluída na letra $\mathrm{C}$.

53 Este vocábulo aparece nos dicionarios da Real Academia Española como prenda feminina. Vid. n. 38. 
FANTASMA: Fantasma. Espectro.

FARTA: Hartazgo.

FARTARSE: Hartarse.

FEDELLO (animal entero): Entero.

FEHEITO: Helecho.

FEIXE (de lagar, la piedra que sirve de peso): Pilón.

FEIXE: Haz.

FENTO: Helecho.

[FERBEDURA: Vid. FERBOR]

FERBER: Hervir.

FERBOR/FERBEDURA: Hervor.

FERBURA: Hervor.

FERRETE (echarlo): Clavetear.

FERRETE (que se hecha a los cordones): Herrete.

FERRO DO MONTE: Barra.

FERROS (en que se ponen las aciones de los estribos): Arriceses ${ }^{54}$.

FESTA: Fiesta.

FESTAS (hacerlas ${ }^{55}$ a los niños, manoseándolos): Popar.

FIBILLA: Hebilla.

FIEL (de puerta, que está para registrar o cobrar): Portalero.

FIESTRA (de aguja): Hondón.

FILAS (de cepas que hai en las viñas): Liños $^{56}$.

FILLASTRO, FILLASTRA: Alnado, Alnada.

FÍO: Hilo.

FÍO (la parte por donde corta el cuchillo, nabaja, etc.): Filo.

FITAR (con riego o cabaduras algún terreno): Amelgar.

FOCHANCO (hecho por las avenidas o corrientes de agua): Barranco. Barranca. Barrancal.

FOFO: Abalado.

FOGAXE (las llagas de los nifios en cara y cabeza): Lactumen.

FOGUEIRA: Hoguera.

FOGUEIRA (que hazen por señal los fachos o otras gentes de atalaia del enemigo): Almenara.

FOL (que trahen los pobres colgado para recoger la limosna): Burjaca.

FOLLA: Hoja.

FOLLA (caída de los árboles): Hojarasca.

FOLLA (de la vid): Pámpana.

\footnotetext{
54 Ms. Atriceses.

${ }^{55}$ o $r$ corrixe un $n$ previo, condicionado por que se, riscado, que fora escrito inicialmente

${ }^{56}$ Ms. linos.
} 
FOLLA (hecharla los árboles): Hojecer.

FONDO (de pipa): Témpano.

FONDOS (de pipa, la rañura en que se aseguran): Xable.

FORMIGA: Hormiga.

FORMIGA (con alas por el otoño): Aluda.

FORNADA: Hornada.

FORNAXE (lo que se da a la hornera): Hornage.

FORNEIRO: Hornero.

FORNILLA: Hornilla.

FORNO: Horno.

FORRA (piedra en que se afianza la palanca): Hipomoclio.

FOSAR: Hocicar. Hozar.

FOSIÑOS: Hocicos.

[FOSTREGA: Vid. FUSTRIGA].

[FOSTREGADA: Vid. FOSTREGAZO].

FOSTREGAZO/FOSTREGADA: Vardascazo.

FOUCE (poda con astil largo para cortar tojos o ramas de árboles): Cimbara.

FOUCIÑO (para cojer ierba, trigo, zenteno, etc.): Dalle.

FOULA (la arina que buela): Harija.

FOUSE (que se pone en un palo para cortar cosas fuertes): Podón ${ }^{57}$.

[FOUSIÑO: Vid. FOUSIÑA].

FOUSIÑA (que es la que tiene dientes como sierra): Hoz.

FOUSIÑA/FOUSIÑO: Hoz. Hocino. Falce.

FRANGULLA: Migaja.

FREBA (la de la carne, jamón, etc., y aquellas hebritas delgadas de ello):

Hebra. Brizna.

FREGAR (lino o otra cosa semexante): Sobar.

FRUNGALLA: Migaja.

FUNGALLA: Migaja.

FUNGUEIRO: Estadoño. Estaca. Estadojo.

FURCO (medida): Xeme.

FUSO: Huso.

FUSTRIGA/FOSTREGA: Vardasca.

GADELLO (de lana o pelo ${ }^{58}$ enrredado): Vedija.

GAFAR (oler mal): Heder.

57 Ms. Podén.

${ }^{58}$ Ms. pello (lapso talvez provocado por Gadello). 
GAITA (que se haze de la ierba alcacer): Avena. Zamponfa ${ }^{59}$. GAIVOTA (ave): Gaviota.

GALADAS (del pescado): Agallas.

GALEADURA (aquella gota del huebo): Galladura.

GALEAR: Gallear.

GALILLO: Gallillo.

GALIÑA: Gallina.

GALO: Gallo.

GANCHO (del candil, o otro de hierro): Garabato.

GANDRA: $\mathrm{Naba}^{60}$.

GARABULLADA (menuda): Támaras.

GARABULLOS (menudos): Támaras.

GARAFETEAR (el cordel con que se haze): Cazumbre.

GARAFETEAR (las vasijas del vino): Cazumbrar.

GARDA (de cruz en la llabe): Rodete.

GARDA (de la llabe): Guarda.

GARDAS (de la llabe, el corte largo que las coge todas, o el paletón enteramente): Rodaplancha.

GARDAS (la parte de la llabe que tiene los cortes): Paletón.

GARFIOS: Garfios.

GARFIOS (en la vid, calabazos, guisantes, etc.): Tijereta.

GARFOS: Garfios.

GATEADO $^{61}$ (lienzo, paño, etc.): Barrado.

GATEAR (echar gatos de hierro): Lañar.

GATEIRA (el sitio a donde se recoje el fuego y ceniza): Hogar.

GATO (animal): Gato.

GATO (de hierro con que se unen ${ }^{62}$ dos piedras o otra cosa): Laña. Grapa.

GAZO (de ubas que se saca del racimo): Carpa.

GOMO (de árbol o planta): Pimpollo.

GORGULLO: Gorgojo.

GORRO (de paño o cosa semejante): Becoquín. Galocha ${ }^{63}$.

GRADAR: Gradar.

GRADE: Grada.

GRAMALLEIRA: Caramilleras.

59

Ms. Zampona.

${ }^{60}$ Entrada repetida en plural.

61 Ms. Geteado. Interpretámolo como erro, aínda que unha forma gueteado sería posíbel por asimilación; mais non parece que Payzal queira en ningún momento reflectir a gheada. O mesmo acontece máis adiante con Leba e trag[u]e.

62 Ms. unas.
${ }^{63}$ Ms. Golocha. 
GRAMAR (el pan): Heñir.

GRAN: Grano.

GRANS: Granos.

GRANS (esparcidos por el suelo): Barreduras.

GRILO (insecto): Grillo.

GRILO (prisión): Brete. Grillete. Grillo.

GRIMO: Grima. Miedo.

GUARDA (de puerta, o que está a la puerta de algún pueblo para registrar o cobrar los derechos): Portalero.

GUERRA (el manifiesto para su declaración): Difidencia.

GURUMELO (que hecha la cebolla, ajo, zenteno, etc.): Tallo.

\section{$[-\mathrm{H}-]$}

HENCHER: Henchir. LLenar.

HENCHER (las fundas): Enfundar.

HICHÓ/HICHOTE (para cojer las perdices vivas): Orzuelo.

HIL]BÁN ${ }^{64}$ : Hi[1]bán. Basta.

HILBANAR: Hilbanar. Bastear.

\section{$[-\mathrm{L}-]$}

LABERCA (pájaro): Cugujada.

LABRADOR (de poca labranza o tierra): Labrantín.

LABREGO: Labriego.

LADRAR (mucho los perros): Ladra.

LADRÓN (aquel sarmiento de la parra que sale del tronco y no de la verdadera vara): Esforrocino.

LAGARTEIRO (ave): Cernícalo.

LAMA: Lodo. Cieno.

LAMA (que queda de las aguas y del profundo de los cimientos de obra): Légamo.

LAMEIRA: Barrizal.

LANDRA: Bellota.

LAREIRA: Hogar.

LARGUEIRO (los palos largos del marco de puerta, bentana o cama): Larguero.

LAZADA: Lazada.

64 A presencia de hilbanar a continuación, tanto en galego como en español, parece xustificar como lapso a ausencia de $l$. 
LEBA E TRAG[U]E ${ }^{65}$ (la persona que lleba y trahe cuentos, chismes y enrredos, y vive de ello): Otacusta.

[LEBANTAMENTO: Vid. LEBANTE]

LEBANTAR A FERBURA: Alzar el hervor.

LEBANTE/LEBANTAMENTO (confusion de gente y voces): Tremolina.

LEBRE: Liebre.

LEBRE (pequeña o nueba): Liebrastón.

LEIRA: Heredad.

LEITARUGA: Chicoria.

LEITUGA: Lechuga.

LEITUGA DOS PORCOS: Chicoria.

LENDIA: Liendre.

LENTO (el fruto o otra cosa que no está mui seca): Liento.

LEÑA (la menuda con que se enciende): Hornija.

LESMA: Babosa.

LESMIÑA: Babosilla.

LIÑA (hilo para coser): Hebra.

LISOS (pieza del telar): Viaderas.

LODOSO (el agua, etc.): Heciento.

LOMBRIGA: Lombriz.

LONDRA: Nutria.

LONTRA: Nutria.

LORDAS (en la capa o en otra cosa): Cazcarrias.

LORDENTO: Cazcarriento.

LOUSA (de barro, como pucheros, etc.; la alacena en que se guarda): Barrera.

LOUSA: Loza.

LUME: Lumbre.

LUME (que hazen por señal los fachos o gente que está de atalaia por causa del enemigo): Almenara.

MACEIRA (en que se da de comer y beber a los animales, y particularmente a los cerdos): Gamella.

MACHORRA: Machorra.

MAINIÑA (la baca u otra qualquiera hembra que no concibe): Machorra. MALLAR (fruto o otra cosa): Sobar.

MANADA (de cerdos): Piara.

${ }^{65}$ Vid. n. 61 
MANDALETEIRO (el que se ejercita en llebar recados y cargas): Ganapán.

MANDIL: Mandil. Ava[n]tal. Zeva[n]tal. Escusalí.

MANOXO: Manojo.

MANTA (peluda): Frazada.

MANTELO (de vestir): Lo mismo que MANDIL.

MARAÑA: Madeja.

MARCAR (la tierra): Alindar.

MARCO (de puerta o bentana, que se forma de largueros y cabios): Marco.

MARRA (de partir piedras): Marra. Almádana.

MARRA (falta que hay en los sembrados): Estar amelgados.

MARRA (por la falta): Marra. Falta.

MARXE (de zenteno, trigo, etc.; la parte vaja o riego): Surco.

MARXE (la parte elevada en que se siembra el trigo, zenteno, etc.): Amelgar.

MARXEAR (hazer los surcos al zenteno, etc.): Amelgar.

MASA (para hazer ostias o vizcochos): Batido.

MASADURA (de golpe que hunde o comprime): Magulladura.

MASADURA (que se eleba por golpe recivido; ve la palabra POTE):

Tolondro. Tolondrón.

MASAR: Sobar.

MASAR (el lino, cáñamo ${ }^{66}$, etc.): Agramar. Macerar.

MASAR (hundiendo o comprimiendo): Magullar.

MASAROCA: Mazorca.

MASAROCA: Mazorca. Husada.

MATERIA (la de las heridas o llagas): Materia. Pus.

MATERIA o SANGRE MASADA: Podre.

MATRÁCOLA: Carraca.

MATULA: Torcida.

MEDA: Hacina.

MEDA (de paja o fruto en ella): Almear ${ }^{67}$. Almiar.

MEDIA (el rollo que se haze de la parte que sobra): Barulé ${ }^{68}$.

MEDICIÑEIRO: Medicastro.

MÉDICO (malo): Medicastro.

MEDIDA (cinta tocada a alguna ymagen): Estadal.

MEDO: Fantasma. Espectro.

${ }^{66}$ Ms. canamo.

67 "Almear, o Almiar" (cfr. Dic. Aut., s.v.)

68 É un galicismo antigo en español, procedente do fr. bas roulé, actualmente virulé. 
MELGACHO: Melgacho. Lixa. Pinta roja.

MELRRO: Mirlo.

MELRRO (de aguas): Pardal.

MERA: Melera.

MERDA: Mierda.

MERDA (de paloma): Palomina.

MERDA (del perro): Canina.

MERDA DE GALIÑA: Gallinaza.

MESTURAR: Mezclar.

MESTURAR (los líquidos): Merar.

METERSE HASTA AS CACHAS, HASTA O PESCOSO, etc.: Meterse hasta las trencas.

MEXOCA: Ampolla.

MINISTRO (gefe de los otros ministros): Barrachel.

MIÑOCA: Lombriz.

MIOLO: Migajón.

MIRAR (con atención y cuidado): Amaitinar.

MÓ: Muela.

MÓ (del molino): Muela. Corredera.

MÓ (que se llama del juicio por nacer tarde): Cordal.

MOCO (el que procede de fluxión y se seca en la nariz como especie de callo): Pólipo.

[MOLIDA: Vid. MOLIDO].

MOLIDO (paño viejo para limpiar): Rodilla.

MOLIDO o MOLIDA (redonda, que se pone debajo del cesto o de otra cosa que se lleba en la cabeza): Rodete.

MONCIÑO (el muchacho de sacristía): Acólito.

MONCIÑO (que lleba el cirial en la yglesia): Ceroferario.

[MONLLA: Vid. MONLLO].

MONLLO/MONLLA (de zenteno, sarmientos, etc.): Gabilla.

MORDEDURA: Tarascada.

MOREA (pescado): Murena.

MORMEIRA (cargazón de cabeza): Azorramiento.

MOROSA: Terrero.

MORTAL (adj., como golpe mortal, etc.): Exicial.

MOSTRA: Muestra.

MOSTRA (que se tiene presente para imitar): Dechado.

MOUCHO (ave): Mochuelo. Alucón.

MUUí̃̃: Molino.

MUÍ̃̃̃ (su pie): Solera.

MULO (por el que es callado y sobre si): Cazurro.

MURCIO (olfato malo de las carnes): Husmo. 
MURO (que divide, cierra o rodea el atrio): Barbacana.

MUSGO: Toba.

$$
[-\mathrm{N}-]
$$

NABALLA: Nabaja.

NABALLA (de punta corba): Falce.

NASA (de mimbres o de hilo): Buitrón.

NATA (de la leche): Tona.

NEBIÑA (especialmente de nabos): Nabina.

NEGRA: Divieso.

NEMBRO (de puerta o bentana): Jamba.

NENTELLA: Faba. Haba.

NESTES: En estos ${ }^{69}$.

NEVIÑA (de rábanos): Rabaniza.

NIÑO: Nido.

NIÑO (en que ponen las gallinas): Ponedero.

NÓ (por nudo): Nudo.

NOGUEIRA (árbol): Nogal.

NOS (fruta): Nuez.

NOVELEIRO (persona que lleba y trahe cuentos, chismes y enrredos, y vive de ello): Otacusta.

NOVELO: Ovillo.

NUDO: Nudo.

$$
[-\mathrm{O}-]
$$

OLLOMOL (pescado): Breca. Breque.

ORRIO: Hörreo.

OSA (animal, la hembra del oso): Osa.

OSIÑO (oso pequeño, el cachorro de la osa): Osesno.

ÓSO: Hueso.

OSO (animal): Oso.

ÓSOS (con que tocan los muchachos): Tarreñas.

OSQUIA (de huso, etc.): Mueca. Muesca.

OSTRA: Ostra.

OSTRÓN: Ostro.

OURISO/OURISO-CACHO: Erizo.

[OURISO-CACHO: Vid. OURISO].

OURO: Oro.

${ }^{69}$ Esta voz aparece na letra $E$, coa entrada en español e a equivalencia en galego. 
OUTEAR: Otear.

OUTEIRO: Otero.

PAA (del horno para hechar el pan): Pala.

PALAMIO: Noque.

PALANCA: Alzaprima.

PALETA (de dar cal): Palustre.

PALETA (la de los canteros de dar cal): Palustre. Paleta.

PALIETARIA (ierba; el dicionario castellano en la voz Alba, haquella del río trahe esta voz, que no está en su lugar): Parietaria ${ }^{70}$.

PALLA: Paja.

PALLA (entera de zenteno u otra cosa): Bálago.

PALLA (la entera o colma de zenteno, la de trigo, etc.): Bálago.

PALLAL: Balagar.

PALLEIRO (de paja entera): Balaguero.

PALLEIRO (de paja o meda): Almear. Almiar.

PALLEIRO (el que se haze de paja): Almear. Almiar.

PALLEIRO (montón de paja): Balaguero.

PALLEIRO (montón de paja, ierba seca, etc.): Balagar. Bálago.

PALMADA (en la cabeza o espaldas): Tamborilada.

PANCHOS (pescado): Fregolino $^{71}$.

PANO (con que se cubre el atril): Atrilera.

PANO (para las babas): Babadero. Babador.

PANO (que se pone a los niños en la cabeza): Gambox.

PANSA ARRIBA (caer, dormir, etc.): De memoria.

PAPO ARRIBA (dormir, caer, etc.): De memoria.

PAREDE: Pared.

PAREDE (que divide, cierra o rodea el atrio): Barbacana.

PASAL (piedra para pasar río o regato): Pasadera. Pasadero.

PASEANTE: Paseante. Azotacalles ${ }^{72}$.

PASEAR (vagando): Barzonear.

PASEO (ocioso): Barzón.

PATA ROXA (pescado): Pinta roja. Lixa.

PAU: Palo.

PAU (en que se raia o señala para contar): Tarja.

PAÙ DE ESTORNELA: Marro.
70 Ms. Paletaria.
71 Vid. n. 37.
72
Ms. Azotacallos. 
PAU DE FERRO: Barra.

PAUS (con que tocan los muchachos): Tarreñas.

PÉ: Pie.

PÉ (de molino): Solera.

PÉ (el bagazo jun[to] en el lagar para esprimirlo): Noque.

PEDIDOR (de limosnas para alguna obra pía): Bacinero. Bacinador. Demandante.

PEDIR (limosna por andar holgando): Gallofear.

PEDOIROS (del tintero): Cendales. Gropos.

PEDOIROS (los del tintero): Algodones.

PEDRA: Piedra.

PEDRA (de los dientes o sarro): Toba.

PEDRA (en que se afianza la palanca): Hipomoclio.

PEDRA (grande separada sobre si): Tormo.

PEDRA DE LAGAR (que sirve de peso para que cargue la viga): Pilón.

[PEDRA MIÚDA: Vid. PEDROGULLO].

PEDRO PÉREZ (la morcilla grande que se haze): Obispillo.

PEDROGULLO/PEDRA MIÚDA (moitas juntas): Rocalla.

PEGADA (estar una leira a otra): Alindar.

PEITUGASO: Pechugón.

PELEXO: Piel.

PELEXO: Pellejo. Cutis.

PELEXO (de entre la pierna y la barriga de la bestia $u$ otro animal): Babilla.

PELOURO: Guija.

PENA (pieza del rodezno del molino): Paleta. Álabe.

PENEIRA (de especies, medicinas, tabaco, etc.): Tamiz $^{73}$.

PESA (la que sirve de peso en la romana): Pilón.

PESADILLA (que se pone de noche sobre el corazón): Yncubo.

PESADO (tomar pesado el vino): Ahilarse.

PESCOSO: Pescuezo.

PESCOSO (del pie): Empeine.

PESCOSÓN: Pestorejón.

PESCOZO (la parte del $^{74}$ buei en que se pone el yugo): Cerviz.

PESTANA: Pestaña.

PESTENEXAR: Pestañear.

PESTILLO: Pestillo.

PETA/PICARAÑA: Piqueta.

PETO (para quartos): Alcancía. Hucha. Olla ciega.

73 Antes desta entrada, aparece o vocábulo peneira, sen equivalencia en español.

${ }^{74}$ A continuación de el. 
PETO (por faltriquera): Faldriquera. Faltriquera. Bolsillo.

PEZCOSO: Pescuezo.

PÍA (de baptismo, el sitio en que está): Baptisterio $^{75}$.

PÍA (en que se da de comer y beber a los animales): Gamella.

PÍA (de fuente): Taza. Pila.

PÍA (grande de fuente): Pilón. Tazón.

[PICARAÑA: Vid. PICA].

PICHETA/PICHEL: Galleta.

[PICHEL: Vid. PICHETA].

PINCERNA: Pertiguero.

PIÑA (de ubas): Racimo.

PÍO (por pila grande de fuente): Pilón. Tazón.

PÍO (de curtir)/PALAMIO (de curtir): Noque.

PIPA (de fumar, la parte en que se le hecha el tabaco): Tabaquera.

PITA CEGA (juego de los muchachos): Gallina ciega.

PITACEGA (que es aquella que anda sobre el agua, de hechura o figura de araña): Tejedera. Escribano del agua.

PLATO (en que se pide limosna): Bacinilla. Bacineta. Bacinica.

PODA (instrumento para podar): Podadera.

POLAINA: Polaina.

POLAINA (la parte de ella que cubre la hebilla): Guardapolvo.

PONTE: Puente.

PONTE (pequeña)/PONTE DE PAÙ/PONTILLÓN: Pontón.

[PONTE DE PAU: Vid. PONTE].

[PONTILLÓN: Vid. PONTE].

POÑEDOR: Ponedor.

POÑEDOR (de medo o grimo): Terrifico.

PONEER: Poner ${ }^{76}$.

PONEER ${ }^{77}$ (gatos de hierro): Lañar.

PORCO: Puerco. Cerdo. Marrano.

PORCO DA CEBA: Cerdo de muerte.

PORCO DE CRÍA: Cerdo de vida.

PORCO ESPIÑO: Erizo.

PORQUERIA: Bascosidad.

PORQUIÑO: Marranillo. Cerdillo. Gorrín.

PORTO DOS CARROS (sitio por donde se pasa el río o regato ${ }^{78}$ ): Vado.

POSO (para enlagar el lino): Embalsadero.

${ }^{75}$ Antes desta entrada aparece Pia de Baptismo sen equivalencia en español

76

Entrada repetida.

77 Ms. Poner.

${ }^{78}$ No ms. etc, riscado. 
POSTIGO: Corredera.

POSTIGO DA PORTA: Corredera. Saetera.

[POSTURA: Vid. PUXA].

POTE (de golpe en la cabeza): Burujón.

POTE (el bulto que se levanta en la cabeza por llebar algún golpe): Porcino.

POTE (que se lebanta por golpe recivido): Chichón.

POTE (que se lebanta por golpe recivido): Tolondro. Tolondrón.

PREMEDEIRA (pieza del telar): Cárcola ${ }^{79}$.

PRENÓSTICO/PRONÓSTICO: Almanak. Pronóstico. Calendario.

PRESA (de agua): Esclusa.

PRESA (en río o regato para sacar agua): Azud. Espaldón. Azut.

PRESUÑA (por la uña que se saca al cerdo o otro animal al tiempo de pelarse): Carnicol.

PRIXEL: Perejil.

[PRONÓSTICO: Vid. PRENÓSTICO].

PULSERA $^{80}$ : Armella. Bracelete.

PUNTEIRA (la vara que se pone en la caña de pescar): Rabiza.

PUNTEIRA (varilla que se pone en la punta de la caña de pescar): Rabisa.

PUÑADO (por lo mismo de las dos manos juntas, etc.): Almorzada. Almosta ${ }^{81}$.

PUÑOS (por las dos manos juntas, llenas de qualquier cosa): Almosta. Almorzada.

PUÑOS o PUÑADO (lo que cabe en las dos manos juntas): Almuerza.

PUXA/POSTURA (que se haze cuando [a]y remates): Puja. Postura. Hacimiento.

$$
[-Q-]
$$

QUEIMOSA: Divieso.

RABADILLA: Rabadilla.

RABADILLA (de las aves): Obispillo.

RABELO: Rabón. Curto.

${ }^{79}$ Entrada repetida.

80 ¿Erro por pulseira?

81 "La cantidad de trigo, ú de otra cosa que puede caber en las dos manos juntas arqueándolas. Es voz antiquada de Aragón, que modernamente se dice Mosta, ó Muesta" (Cfr. Dic. Aut., s.v.). 
RABO (de pera u otra fruta): Pezón.

RABUÑAR: Arañar. Arpar.

RACHAR (hazer tiras o pedazos ropa, etc.): Rasgar. Arpar.

RAIA (aspa o testadura de lo escrito): Tachón.

RAIA (que se pone al fin del renglón, quando queda dividida o por acabar la voz): División.

RAIAR (aspar o testar lo escrito): Tachar.

RAIIZ: Raíz.

RAİZ (de árbol que queda después de cortado): Tocón ${ }^{82}$.

RAMALLOSA (que sirve de muro): Seto.

RAMOS (el muro que se haze con ellos): Seto.

RANCEARSE: Columpiarse.

RANCEHEIRA: Columpio.

RANDEARSE: Columpiarse.

RANDEHEIRA: Columpio.

[RASCADOR: Vid. RASCÓN].

RASCAR (limpiar las caballerías): Almohazar.

RASCÓN (con que se limpian las caballerías): Almohaza.

RASCÓN (de hierro para limpiar las caballerías): A[l]mohaza.

RASCÓN (de limpiar las caballerías): Almoaza.

RASCÓN/RASCADOR (cepillo redondo de cerdas para limpiar los caballos): Bruza.

RASPA: Badil.

RASTRO (en que se lleba piedra, madera, etc.): Narria. Mierra.

RASTRO (en que se lleban piedras o otras cosas): Narria. Mierra.

RAXO DE PORCO (o de otro animal): Yjada.

REBEIRAS (lo que se saca al trigo o zenteno quando se limpia): Acribaduras.

REBOLA: Rasero.

REBOLTO: Rebuelto. Heciento.

RECHONCHO (hombre pequeño y gordo): Recoquín.

REFOLLO (por las bueltas que haze el aire): Vórtice.

REFOLLOSO (por el remolino que haze el aire o el agua): Vortiginoso.

REGATEADURA (compra de huebos, gallinas, etc., que se haze para bolber a vender): Recova.

REGATEIRA (que compra por los lugares): Recovera.

REGO (el surco que se haze en los zentenos, trigos, etc.): Surco.

REGO (que hazen los aguaceros o corrientes de aguas): Badén.

RELICARIO: Patena.

REMACHAR (las puntas de los clabos): Robrar.

${ }^{82}$ Ms. Tacón. 
REMUIĨ̃̃: Remolino.

REMUÍÑO: Vórtice.

REMUÍÑO: Remolino. Vórtice.

REMUÍÑO (de polbo): Remolino. Tolvanera. Vórtice.

REMUIÑOSO (por el remolino que haze la agua o aire): Vortiginoso.

RENDER (como la linaza, el vino, etc. [..], o sale más de lo que se pensaba): Cundir.

REPINICOQUES (adornos en las empanadas o pasteles): Repulgo

REQUESTA (tomar satisfacción): Recombención.

RESCALDO: Rescoldo.

RESPIGO: Respigón. Padrast[r]o.

RESPIGO (el pellejo que se levanta junto a las uñas): Padrast[r]o.

RESTA o RESTRA: Ristra.

RESTELAR: Rastrillar.

RESTELO: Rastrillo.

[RESTRA: Vid. RESTA].

RESTROLLO (lo inferior y duro de la paja): Tramojo.

RETELLAR: Tejar.

RETRANCA: Ataharre.

REVEIRAS (las escabaduras o limpias del zenteno, etc.): Granzas.

REXÓN: Chicharrón.

RIFAR: Reñir.

RIPA: Ripia.

ROCA (para hilar): Rueca.

RODEIRA: Carril.

RODEIRA: Rodada.

RODEIRA (de carro): Rodada.

RODICIO: Rodezno.

RODUCIO: Rodezno.

ROLETE (palos pequeños sobre que se lleban cosas de peso rodando): Rodillo.

ROLLO (que se haze de la media que sobra): Barule $^{83}$.

ROMANA (la pesa que en ella sirve para señalar el peso): Pilón.

ROQUEIRO (con que se asegura lo que se hila): Cucurucho.

ROSADURA: Colisión.

ROXELO:

RUTA: Rauta. 
[SÁBALO: Vid. SABLE].

SABÁN: Sábana.

SÁBENA: Sábana.

SÁBENA (o otra cosa que sirve de cortina a la puerta de la taberna): Carpeta.

SABENLA (pescado): Saboga.

SABLE o SÁBALO (pescado): Sábalo.

SABUGUEIRO: Saúco.

SACAR O CALDO: Escudillar.

SAMBESUGAS: Sanguijuelas.

SAMBIJUELAS: Sanguijuelas.

SAMBORCA (pescado): Saboga.

SAMURDO (por el que es callado y sobre si): Cazurro.

SANGRE MASADA: Podre.

SAPATO (el pedazo que cae sobre el empeine): Polaca ${ }^{84}$.

SARAMAGO: Xaramago.

SARRIO: Sarro.

SARRIO (de los dientes): Toba.

SEÑAL (de cita en los libros): Asterisco.

SEÑALAR (algún terreno con cabaduras o riego): Amelgar.

SEO: Seno.

SEPO (para picar): Tajo. Picador.

SERDA (la fuerte y aguda del cerdo): Porcipelo.

SILBA: Zarza.

SILBAS (que se ponen en los muros para defenderlos): Barda.

SISCO (que se barre o limpia): Barreduras.

SISCO (y todo lo más que se barre): Basura.

SITIO (en que está la pila baptismal): Baptisterio.

SOBREIRA: Alcornoque.

SOBRELUME: Dintel.

SOBRETODO: Escrocón. Casacón. Sobrevesta.

SOCALCO: Espolón.

SOCALCOS (de tierra): Graderías. Bancales.

SOCO: Zueco. Galocha. Haloza ${ }^{85}$.

SOCOS (zapatos de palo): Galochas.

SOGRA: Suegra. Madre política.

84 "El copete, ò vuelta del zapato, que cae sobre el empéine del pié, y suele ser de diferente color" (cfr. Dic. Aut., s.v.).

${ }^{85}$ A cotinuación aparece ve. a N. verbo Gallica. 
SOGRO: Suegro. Padre político.

SOLEIRA: Solera.

SOPLILLO: Buxeta.

SOPLILLO: Tabaquera.

SUME (el agua o licor de árbol, planta, fruta, etc.): Jugo.

SURCIR (tapar los a[g]ujeros o claros, pasando hilos): Corcusir ${ }^{86}$.

\section{$[-\mathrm{U}-]$}

UÑA (de cerdo o otro animal de pata hendida que se le saca al pelarse):

Carnicol.

\section{$[-\mathrm{V}-]$}

VAGAMUNDO: Vago. Baldío.

VAGAMUNDO (el que anda pidiendo por holgar): Gallofero.

VENENO (por la materia de las llagas o heridas): Pus. Materia.

VERME: Gusano.

VERTEDEIRO (de innundicias): Albañal.

VIDRO: Vidrio.

VIDRO (el de cada luna de los anteojos): Espejuelo.

VINAXEIRA: Vinagera. Ampolla. Ampolleta.

VIÑA (el pie de ella): Vid.

VIÑA ALTA ( o emparrada): Parra.

VIÑA BRABA (silbestre): Labrusca.

VIVOS (puntadas en el colchón): Bastas.

XA: Ya.

XABRÓN: Xabón.

XAMBA (de puerta o bentana): Jamba.

XAMÚAS: Xamugas.

XAQUETE $^{87}$ (juego): Tabla.

XASTRE: Sastre.

XERRO (picheta con vico): Galleta.

XO (voz con que se espantan las gallinas y aves): Ox.

XUANIÑA (insecto redondo encarnado): Casita de Dios ${ }^{88}$.

${ }^{86}$ Ms. Concursir, repetido por un borrón de tinta sobre o primeiro.

${ }^{87}$ Vid. n. 38. 
XUFRE: Azufre.

XUGO (con que se uncen los bueies): Yugo.

XUGO (la parte de el en que se pone el pescuezo): Camella.

XUGO (sumo de árbol, planta, fruta, etc.): Jugo.

XUGUEIRA (la parte del pescuezo del buei a donde se le pone el yugo): Cerviz.

XUNTOIRO: Perpiaño.

\section{$[-\mathrm{Y}-]$}

YCHÓ/YCHOTE (armadilla para cojer vivas las perdizes): Orzuelo.

[YCHOTE: Vid. YCHÓ].

YLEIRA: Hilera.

YLEIRA (de cepas que hai en las viñas): Liños.

88 "Insecto pequefio como medio guisante, de color encamado, que vuela poco; pero no hace daño, y se cria en el campo entre la immundicia. Llámanle assi los niños, porque la figúra es como de una media esphéra, y quando quiere volar parece al abrir las alas que abre unas puertas" (cfr. Dic. Aut., s.v. CASA). 